\title{
A Rare Cause of Dyspnea: Undifferentiated Pleomorphic Sarcoma in the Left Atrium
}

\author{
Martin A. Munin, f, Maria Silvia Goerner ${ }^{\mathrm{a}}$, Ignacio Raggio ${ }^{\mathrm{a}}$, Jorge Wisner ${ }^{\mathrm{b}}$, Alejandro Tettamanzi ${ }^{\mathrm{b}}$, \\ Jose Godiac $^{c}$, Maria Paula Penaloza ${ }^{\mathrm{d}}$, Javier Guetta ${ }^{\mathrm{e}}$
}

\begin{abstract}
Primary cardiac tumors are a rare entity. The presence of a mass in the left atrium suggests myxoma as a first differential diagnosis. Here we present a rare case of a woman with exertional dyspnea with a large tumor in the left atrium. The patient was extensively studied with echocardiography, cardiac MRI, coronary angiography and computed tomography. Extracardiac neoplastic involvement was ruled out. The patient underwent surgery, the mass was removed, and the final diagnosis was an undifferentiated pleomorphic sarcoma.
\end{abstract}

Keywords: Auricular mass; Atrial tumor; Undifferentiated sarcoma

\section{Introduction}

A middle-aged woman with no history of cardiovascular disease consulted the hospital referring exertional dyspnea. The patient was extensively studied. The transthoracic echocardiogram showed a big heterogeneous multi-lobed mass, highly mobile, attached to the posterolateral atrial wall prolapsing into the left ventricle during diastole. The tumor produced functional mitral valve stenosis, the transvalvular mean gradient was $8 \mathrm{~mm} \mathrm{Hg}$ and pulmonary systolic artery pressure was $45 \mathrm{~mm} \mathrm{Hg}$. The right and left ventricular systolic func-

Manuscript submitted August 11, 2017, accepted August 28, 2017

aDepartment of Echocardiography, CEMIC Hospital, Buenos Aires, Argentina. Av. E Galvan 4102, C1431FWO, Buenos Aires, Argentina

bepartment of Catheterization and Cardiovascular Interventions, CEMIC Hospital, Buenos Aires, Argentina. Av. E Galvan 4102, C1431FWO, Buenos Aires, Argentina

${ }^{\mathrm{c} D e p a r t m e n t}$ of Cardiovascular Surgery, CEMIC Hospital, Buenos Aires, Argentina. Av. E Galvan 4102, C1431FWO, Buenos Aires, Argentina

dDepartment of Pathology, CEMIC Hospital, Buenos Aires, Argentina. Av. E Galvan 4102, C1431FWO, Buenos Aires, Argentina

eDepartment of Cardiology, CEMIC Hospital, Buenos Aires, Argentina. Av. E Galvan 4102, C1431FWO, Buenos Aires, Argentina

CEMIC Hospital, Buenos Aires, Argentina. Av. E Galvan 4102, C1431FWO, Buenos Aires, Argentina

fCorresponding Author: Martin A. Munin, Department of Echocardiography, CEMIC Hospital, Buenos Aires, Argentina. Av. E Galvan 4102, C1431FWO,

Buenos Aires, Argentina. Email: mmunin@gmail.com

doi: https://doi.org/10.14740/cr590w tion was normal. The workup continued with transesophageal echocardiogram and cardiac MRI that confirmed the findings and showed another mass in the anterior atrial aspect. Imaging studies suggested a malignant nature of the tumors. Afterwards a thoraco-abdominopelvic CT ruled out extracardiac tumor involvement. The patient underwent surgery, the masses were extensively infiltrating the left atrial wall and could not be completely removed. The pathological samples revealed an undifferentiated pleomorphic sarcoma (malignant fibrous histiocytoma).

\section{Case Report}

We report a case of a 52-year-old woman with a large left atrial tumor. The patient complained of 2 months of exertional dyspnea. She had no history of cardiovascular disease, and denied having fever or weight loss lately.

At the time of consultation, she had blood pressure of 130/80 $\mathrm{mm} \mathrm{Hg}$, a heart rate of $74 \mathrm{bpm}$, respiratory rate of 20 breaths per minute, temperature of $36.2{ }^{\circ} \mathrm{C}$, and oxygen saturation of $99 \%$ on room air. No remarkable signs were found on physical examination. The results of laboratory tests showed a hematocrit of $35 \%$ without other significant data. The electrocardiogram was normal. Chest radiography showed a slight redistribution of flow to the vertices without other pathologic findings.

The patient was studied with transthoracic Doppler echocardiogram. The study showed preserved systolic left and right ventricular function. A big heterogeneous multi-lobed mass, highly mobile, attached to the posterolateral atrial wall, was observed (Fig. 1a). The tumor prolapsed through the mitral valve into the left ventricle in diastole producing functional mitral valve stenosis. Mitral transvalvular mean gradient was estimated in $8 \mathrm{~mm} \mathrm{Hg}$. Pulmonary systolic artery pressure was $45 \mathrm{~mm} \mathrm{Hg}$. Afterwards, the examination continued with a transesophageal echo. The study showed a huge multi-lobed auricular mass, $67 \mathrm{~mm}$ in length, with a large base of implantation to the posterolateral aspect of the left atrial wall (Fig. $1 b)$. Another small sessile mass was observed in the anterior surface of the left atrium (Fig. 2). Pulmonary veins were not affected.

In order to better investigate the disease, a cardiac MRI was performed. The study showed again two masses in the left atrium. The larger multi-lobed mass, measuring $64 \times 19 \times 18$ $\mathrm{mm}$, was seen prolapsing into the left ventricle during diastole. Of note, the tumor was widely attached to the posterolat- 


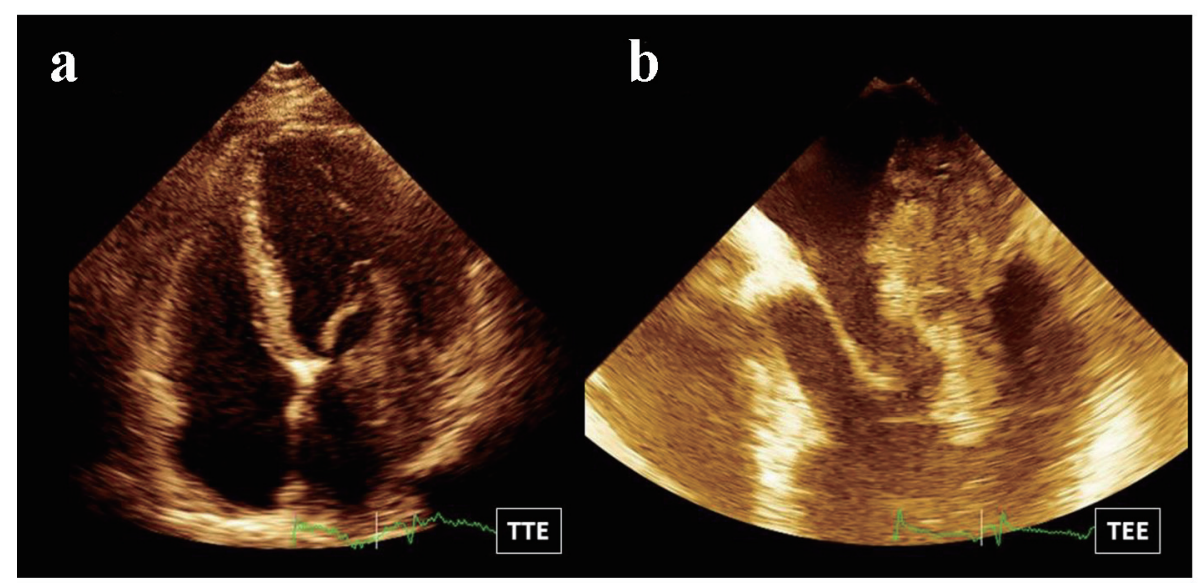

Figure 1. (a) Four-chamber view: there is a big heterogeneous multi-lobed mass attached to the posterolateral atrial wall. (b) Transesophageal echo: a huge multi-lobed auricular mass with a large base of implantation to the posterolateral aspect of the left atrial wall is observed.

eral wall of the left atrium. Moreover, the other sessile mass in the anterior wall contiguous with the rear wall of the aorta measuring $13 \times 5 \times 5 \mathrm{~mm}$ was observed (Fig. 3). Both tumors were isointense in $\mathrm{T} 1$ and hyperintense on T2 and T2 STIR. No contrast enhancement was observed in the images of the first phase (sequence of perfusion in real time). Delayed contrast enhancement imaging revealed heterogeneous hyperenhancement of the mass (Fig. 4).

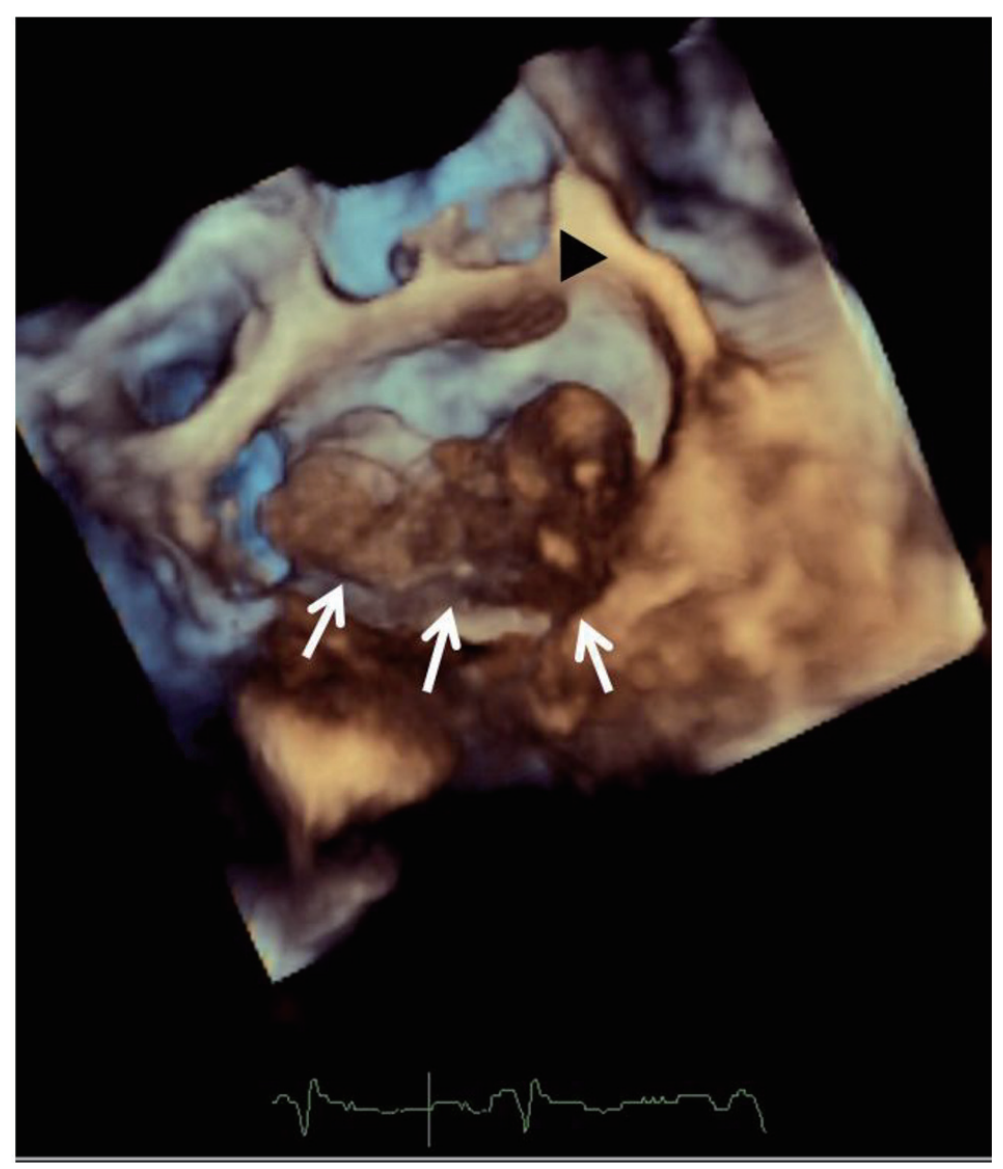

Figure 2. Transesophageal echocardiography: a 3D zoom mode image view from the left atrium shows a huge multi-lobed auricular mass with a large base of implantation to the posterolateral aspect of the left atrial wall, white arrows. A small sessile mass is seen on the anterior aspect of the atrium, black arrow head. 


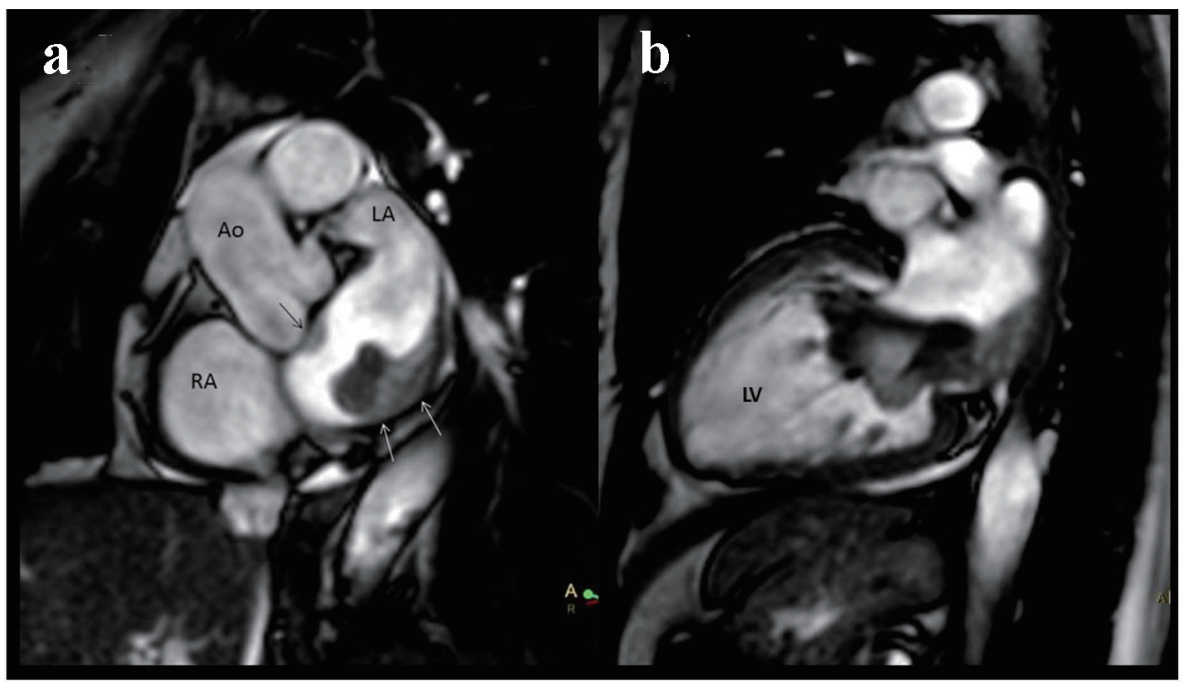

Figure 3. Cardiac MRI. (a) The tumor was widely attached to the posterolateral wall of the left atrium, white arrows. Another sessile mass in the anterior wall of the left atrium is seen, black arrow. (b) Two-chamber view. The atrial tumor prolapses into the left ventricle during diastole.

The results of echocardiography and MRI suggested that tumors could be malignant. Then, a thoraco abdominopelvic CT was done which ruled out extracardiac tumor involvement. It was decided to perform heart surgery. Preoperative coronary angiography showed no coronary artery disease. There was an extensive tumor perfusion through a branch of the right coronary artery.

At surgery, the presence of two left atrial masses was con- firmed. The larger mass was very vascularized and had a broad base of implantation to the posterolateral left atrial surface. The tumor was infiltrating the wall and could not be removed completely (Fig. 5). The smaller tumor was also resected. The pathological samples later revealed an undifferentiated pleomorphic sarcoma (malignant fibrous histiocytoma) (Fig. 6).

The postoperative period progressed favorably. The patient was discharged with advice for continuation at the oncol-

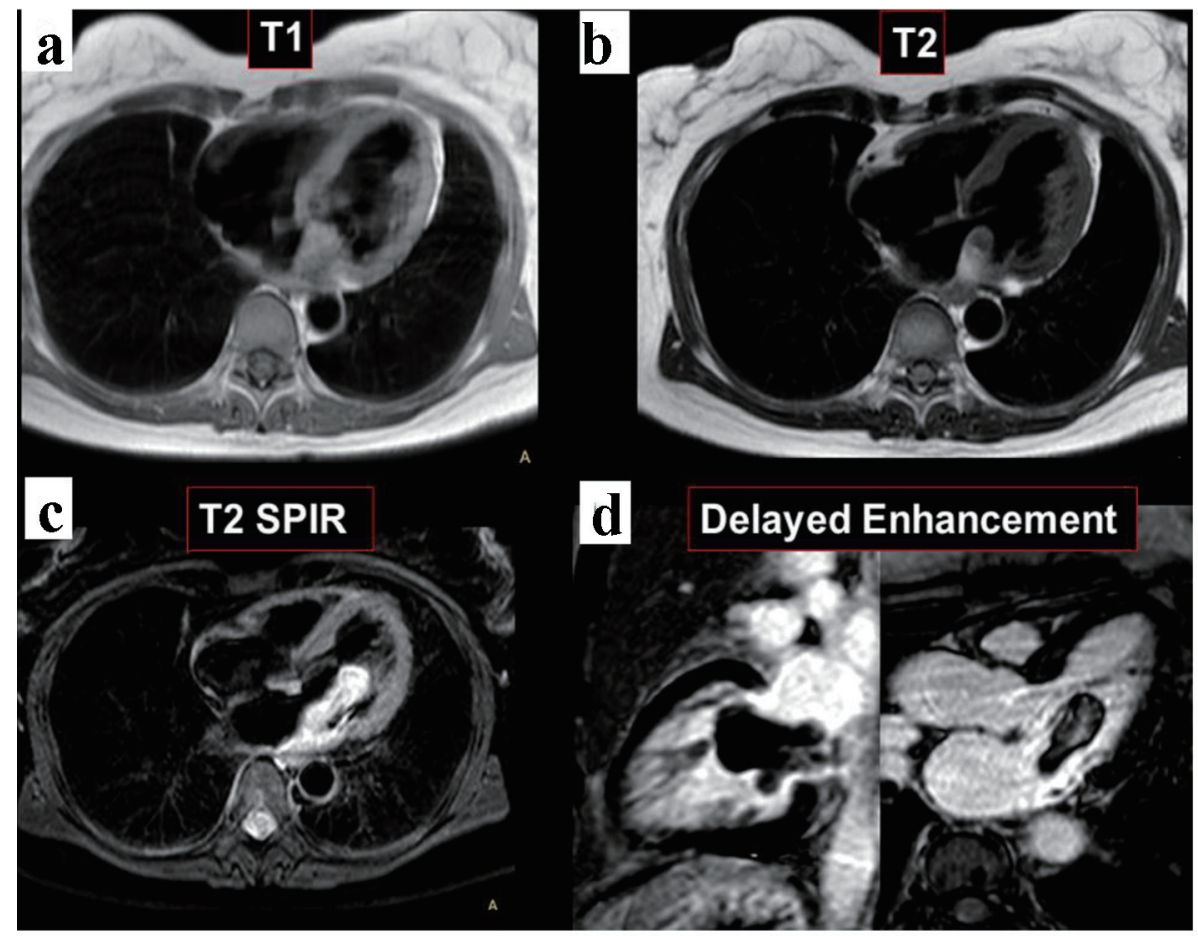

Figure 4. Cardiac MRI. Both tumors are isointense in T1-weighted black blood imaging (a). The masses are hyperintense on T2 and T2 STIR sequences (b, c). Delayed enhancement imaging exposed heterogeneous hyperenhancement of the mass (d). 


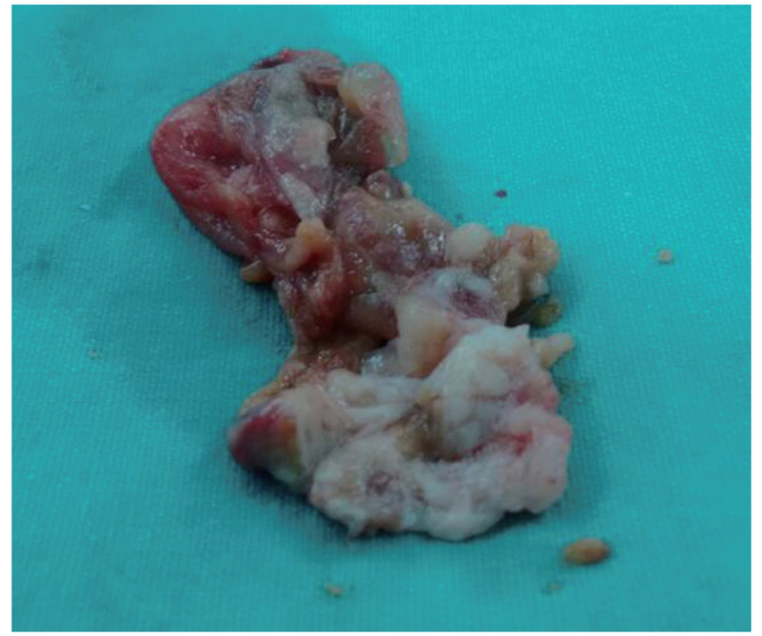

Figure 5. A photograph of the surgical specimen. The mass was heterogeneous and very vascularized.

ogy visit for evaluation of therapy options.

\section{Discussion}

Cardiac tumors, in general, are rare. Metastatic involvement of the heart is $100-500$ times more frequent than primary cardiac tumors [1]. The incidence of primary cardiac tumors is extremely low, ranging from $0.0017 \%$ to $0.019 \%$ [2]. Of these, about three-quarters are benign, commonly myxomas, and the remaining quarter is formed by malignant tumors that are predominantly sarcomas $[3,4]$.

The finding of a left atrial mass often suggests myxoma as the first diagnosis. The differential diagnosis with a malignant tumor is not easy. Of note, when left atrial masses (benign or malignant) cause symptoms, the most common presentation is dyspnea due to obstruction of the mitral valve function $[5,6]$. The characteristic findings that can aid in differential diagnosis of sarcomas from myxomas are: 1) non-septal attachment of mass, 2) multiple masses, 3) broad attachment on the left atrial wall, 4) extension into the pulmonary vein, and 5) semisolid consistency [7]. Many of these features were present in our case.

Cardiac MRI, through many sequences used for tissue characterization, can be helpful for the distinction of benign versus malignant neoplasms [8]. Of course this is not always possible, as in this case. The CMR was valuable to corroborate the presence of two atrial masses, to determine its volume, and to confirm that the larger tumor had a broad base of implantation to the posterolateral wall, all findings suggestive of malignancy. However, the results of T1, T2, T2 STIR, perfusion and late gadolinium enhancement sequences were not useful to define the malignant nature of the mass.

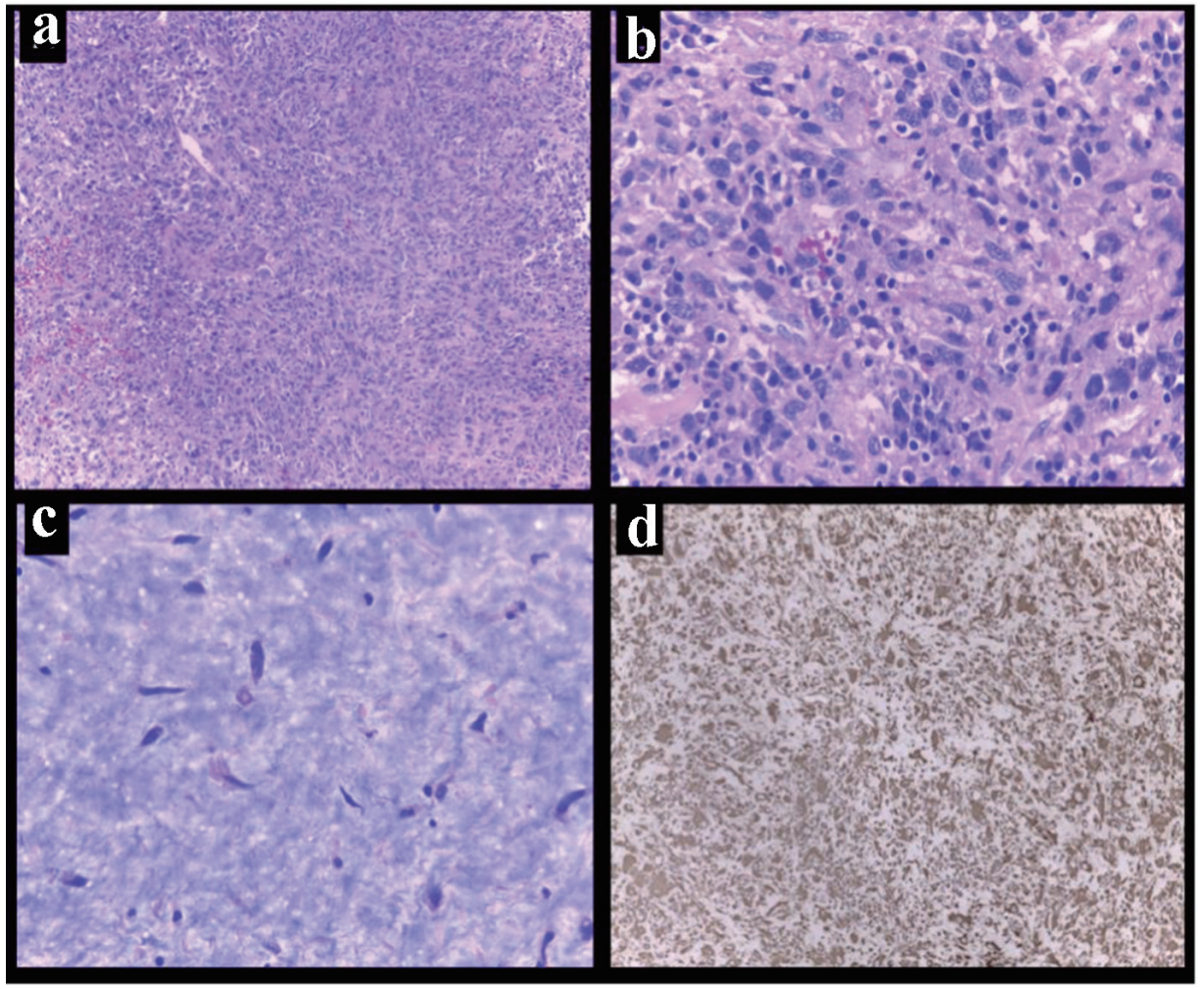

Figure 6. Primary cardiac undifferentiated pleomorphic sarcoma. (a) Low-power magnification showed hypercellular lesion. (b) High-power magnification recognized cells with marked nuclear pleomorphism and cytologic atypia in fibrous stroma. (c) In other areas, hypocellular lesion in myxoid background (hematoxylin and eosin). (d) Immunoreactivity for vimentin in cell showing diffuse positivity (vimentin, D9). 
The prognosis of cardiac sarcomas is pretty bad, with median survival of less than 1 year.

The best therapy for patients with cardiac sarcoma without metastasis is a complete surgical resection of the tumor. Unfortunately, a complete surgical resection is often not possible due to the broaden invasion of cardiac structures. If complete surgical tumor removal is not feasible, more than $90 \%$ of patients die within 1year despite any postsurgical therapy [9].

The effectiveness of chemotherapy and radiotherapy remains undefined [10].

The cardiac transplantation could be an option to consider.

\section{Conflict of Interest}

The authors declare that they have no conflict of interest.

\section{References}

1. Chiles C, Woodard PK, Gutierrez FR, Link KM. Metastatic involvement of the heart and pericardium: CT and MR imaging. Radiographics. 2001;21(2):439-449.

2. Straus R, Merliss R. Primary tumor of the heart. Arch Pathol. 1945;39:74-78.

3. Reardon, Smythe. Cardiac neoplasms. Cardiac surgery in the adult. Edited by: Edmunds LH Jr. McGrawHill: New York, NY. 2003; p. 1373-1400.

4. Mc Allister H, Fenoglio J. Tumors of cardiovascular system in: Atlas of tumor pathology, series 2. Armed Forces Institute of Pathology: Washington, DC. 1978.

5. Fisicaro A, Slavich M, Agricola E, Marini C, Margonato A. Acute pulmonary edema caused by a giant atrial myxoma. Case Rep Med. 2013;2013:904952.

6. Pergolini A, Zampi G, Sbaraglia F, Musumeci F. Left atrial myxoma: two sides of the same coin. J Cardiovasc Med (Hagerstown). 2015;16(Suppl 2):S74-76.

7. Kim JT, Baek WK, Kim KH, Yoon YH, Kim DH, Lim HK. A primary cardiac sarcoma preoperatively presented as a benign left atrial myxoma. Yonsei Med J. 2003;44(3):530-533.

8. Pazos-Lopez P, Pozo E, Siqueira ME, Garcia-Lunar I, Cham M, Jacobi A, Macaluso F, et al. Value of CMR for the differential diagnosis of cardiac masses. JACC Cardiovasc Imaging. 2014;7(9):896-905.

9. Orlandi A, Ferlosio A, Roselli M, Chiariello L, Spagnoli LG. Cardiac sarcomas: an update. J Thorac Oncol. 2010;5(9):1483-1489.

10. Llombart-Cussac A, Pivot X, Contesso G, Rhor-Alvarado A, Delord JP, Spielmann M, Tursz T, et al. Adjuvant chemotherapy for primary cardiac sarcomas: the IGR experience. Br J Cancer. 1998;78(12):1624-1628. 